\title{
Differences in bone mineral density of trajectory between lumbar cortical and traditional pedicle screws
}

Renjie Zhang ${ }^{1,3+}$, Hai Gao ${ }^{2,3 \dagger}$, Huimin Li ${ }^{1,3+}$, Tao Xing ${ }^{1,3}$, Chongyu Jia ${ }^{1,3}$, Jianxiang Zhang ${ }^{1,3}$, Fulong Dong ${ }^{1,3}$ and Cailiang Shen ${ }^{1,3^{*}}$ (D)

\begin{abstract}
Background: Cortical bone trajectory (CBT) has been well-known in spine surgery for obtaining improved fixation while minimizing soft tissue dissection. This study was designed to compare the bone mineral density (BMD) between the CBT and traditional trajectory (TT) by using Hounsfield unit (HU) values and identify the ideal decades of patients and the suitable lumbar segments using this CBT technology from a radiological standpoint.

Methods: Patients were selected randomly from an institutional database based on age (evenly distributed by a decade of life) and gender. A total of 240 healthy patients had a computed tomography (CT) scan of the chest, abdomen, and pelvis. For each patient, axial slices of every vertebra were cut in two planes: one horizontal to the pedicle representing the plane wherein pedicle screws were inserted using the $\Pi$ and the other in a caudocranial plane representing the plane wherein pedicle screws were inserted using the CBT. For each trajectory, a region of interest (ROI) was selected within the area wherein the screws were inserted. A CT number (HU values) was then calculated within each $\mathrm{ROI}$ to represent bone density.

Results: $\mathrm{HU}$ values measured at the ROI of CBT were significantly greater than those of the traditional pedicle screw in all age groups, and the specific value (ratio of the $\mathrm{HU}$ values of CBT/the HU values of TT) between CBT and $\Pi$ was 1.92. A significant difference was observed between male and female. The HU values of CBT and $T$ of males were generally higher than those of females (males: CBT/TT $1.89 \pm 0.45$; Females: CBT/TT $1.95 \pm 0.47$ ). The specific value in HU values significantly increased with increasing age $(p=0.000)$ and cauda lumbar level $(p=0$. 000) in males and females.
\end{abstract}

Conclusion: $\mathrm{BMD}$, as measured by $\mathrm{HU}$ values for the ROI of the CBT screw, was significantly greater than that of the traditional pedicle screw, especially in old patients and cauda lumbar segments.

Keywords: Cortical bone trajectory, Radiological study, CT number, Hounsfield unit

\footnotetext{
*Correspondence: shencailiang1616@163.com

${ }^{\dagger}$ Renjie Zhang, Hai Gao and Huimin Li contributed equally to the study as the co-first authors.

'Department of Orthopedics, The First Affiliated Hospital of Anhui Medical University, 210 Jixi Road, Hefei 230022, Anhui, China

${ }^{3}$ Department of Orthopedics and Spine Surgery, The First Affiliated Hospital

of Anhui Medical University, Hefei 230022, Anhui, China

Full list of author information is available at the end of the article
}

(c) The Author(s). 2019 Open Access This article is distributed under the terms of the Creative Commons Attribution 4.0 International License (http://creativecommons.org/licenses/by/4.0/), which permits unrestricted use, distribution, and reproduction in any medium, provided you give appropriate credit to the original author(s) and the source, provide a link to the Creative Commons license, and indicate if changes were made. The Creative Commons Public Domain Dedication waiver (http://creativecommons.org/publicdomain/zero/1.0/) applies to the data made available in this article, unless otherwise stated. 


\section{Introduction}

Lumbar pedicle screw instrumentation has been a prop clinical surgeon technique for spinal fusion in treating a wide range of spinal disorders, such as fracture, degenerative diseases, scoliosis, kyphotic deformities, and tumors. Traditional pedicle screw fixation provides numerous advantages, the most important of which is reducing rates of loss of fixation and nonunion [1]; however, screw loosening and loss of stability of construct also occur, particularly in patients with poor bone quality [2]. Many laboratory investigations have consistently shown that osteoporotic vertebrae demonstrate reduced insertion torque and pullout strength [3-6]. To solve this challenge, an alternative to traditional pedicle screw trajectory fixation has recently been offered in lumbar fusion constructs. Cortical bone trajectory (CBT) utilizes a medialized trajectory in the caudocephalad direction to engage the cortical bone associated with pars interarticularis and pedicle [7]. The CBT technique has a theoretical benefit for patients with osteoporosis because cortical bone is less affected by the osteoporotic process than cancellous bone. A biomechanical study of CBT has revealed a $30 \%$ increase in resistance to axial pullout load compared with that in the traditional trajectory (TT) in osteoporotic cadaveric lumbar spine [7]. As a common factor used to predict fixation strength, the insertional torque using the CBT technique is $71 \%$ higher than that of the traditional technique in terms of mechanical behavior in vivo [8]. Baluch et al. [9] also examined the fixation strength of CBT screws under cyclic physiological loads and demonstrated superior resistance to craniocaudal toggling compared with that of traditional pedicle screws.

Computed tomography (CT) has become increasingly important in the field of orthopedic research in estimating bone strength, and its application in diagnosing osteoporosis and assessing fracture risks is being investigated. CT numbers, expressed in Hounsfield units (HUs), can be used to calculate bone mineral density (BMD) and estimate bone strength [10-12]. HUs have also been used to approximate regional oral BMD and have been shown to correlate strongly with insertion torque and the stability of metal implants in vitro and in vivo [13-15]. The aims of this study are to evaluate such findings from a radiological standpoint by comparing the average bone $\mathrm{CT}$ number (HU values) within the area wherein pedicle screws are normally placed for both trajectories and determine the optimum ages and lumbar segments.

\section{Materials and methods}

Between December 2015 and May 2017, all healthy patients who underwent consecutive physical examinations had a CT (GE, Discovery CT 750 HD) scan of the chest, abdomen, and pelvis. Reformatted images with a thickness of $0.625 \mathrm{~mm}$ were obtained. Scan parameters included $120 \mathrm{kV}, 440 \mathrm{~mA}$, a $512 \times$ 512 matrix, a layer thickness of $0.6 \mathrm{~mm}$, and a pitch of $0.6 \mathrm{~mm}$. Two-dimensional reconstructions were obtained in the coronal and sagittal planes. Patients were excluded if they had a spine fracture, history of spine surgery, infection, tumor, deformity, and obvious hyperostosis of the facet joint. Patients less than 20 years of age or patients with a width of pedicle less than the region of interest (ROI) were also excluded. A total of 240 patients were randomly selected for inclusion based on age and gender. Forty patients (20 of each gender) in each decade of life (from age 20 to above 70) were examined. An ROI was selected using axial slices of the lumbar vertebrae of patients for each trajectory by using GE picture archiving and communication system (General Electric Medical Systems, Milwaukee, WI, USA) to calculate an average $\mathrm{HU}$ value. Protocols and cadavers were used in accordance with the ethical guidelines established by the local ethics committee and were approved by the institutional review board (IRB) of the author's affiliated institutions.

For the TT, the axial CT images were sliced in a plane horizontal to the pedicle. The ROI was fixed at $40 \mathrm{~mm} \times$ $6.0 \mathrm{~mm}$ [16], which represented the pedicle screws used in the TT, and the entry point was set at the bisection of a vertical line through the facet joints and a horizontal line through the transverse process (Fig. 1a). The ROI started at this entry point and was directed toward the pedicle midline to represent the ideal area wherein to insert the screws (Fig. 1b).

For the CBT, the axial images were sliced in a caudocranial plane. The ROI was fixed at $30 \mathrm{~mm} \times 4.0 \mathrm{~mm}$ (16), which represented the small and short pedicle screws used in the CBT. The starting point was confirmed to be the junction of the center of the superior articular process and $1 \mathrm{~mm}$ inferior to the inferior border of the transverse process (Fig. 1c). The ROI started at this entry point and was directed toward the pedicle midline to represent the ideal area wherein to insert the screws (Fig. 1d).

All measurements in our study were performed by two spine surgeons who were familiar with anatomy and the CBT screw insertion technique in the lumbar spine (L1L5). Each parameter was measured thrice in each vertebra by the spine surgeons. The mean values were obtained to be the ultimate value.

\section{Statistical analysis}

All statistics were calculated using SPSS software version 16.0 (IBM Corporation, Armonk, NY, USA). Descriptive statistics, including frequency and mean \pm standard 

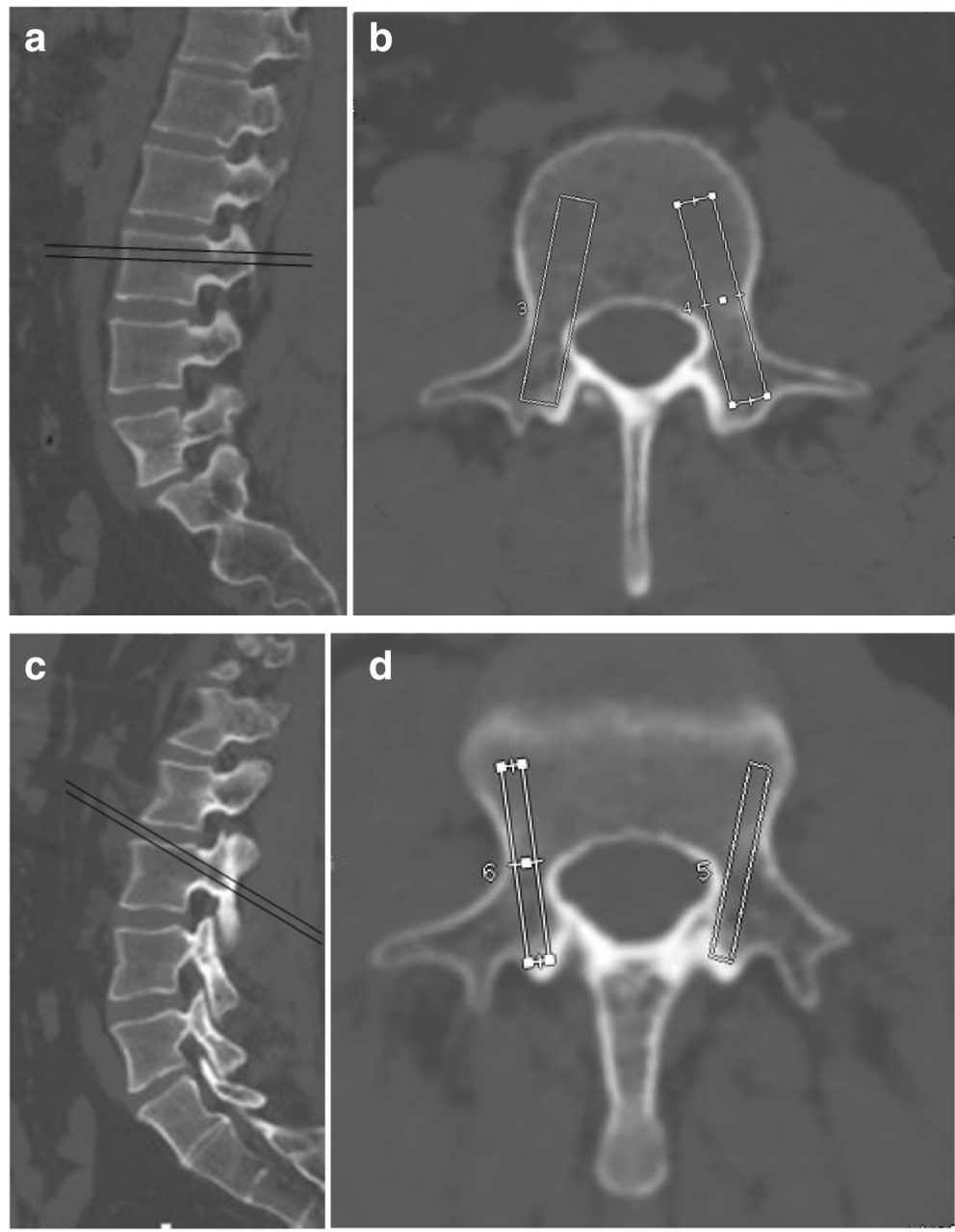

Fig. 1 Sagittal thin-sliced CT images displaying the screw insertion angle for the $\Pi$ (a) and CBT (c). ROI used for calculating the CT numbers for the $\Pi(\mathbf{b})$ and $C B T(\mathbf{d})$

deviation (s.d.), were calculated. Paired $t$ tests were used to compare paired variables between left and right pedicles and CBT and TT. The one-way test was used to compare the measured $\mathrm{HU}$ values among different decades and lumbar segments. Correlations between $\mathrm{HU}$ values and age and segment were calculated by using the Pearson correlation coefficient. Statistical significance was defined as $p<0.05$.

\section{Results}

The detailed information about patients' demographics is shown in Table 1. No significant difference was determined between the $\mathrm{HU}$ values at the ROI of the CBT and TT of left and right pedicles (CBT: $p=0.053, t$ $=1.935$; TT: $p=0.474, t=0.716$ ). The mean $\mathrm{HU}$ values were $365.19 \pm 105.05$ by CBT and $199.02 \pm$ 67.89 by TT (Table 2). The specific value (ratio of the $\mathrm{HU}$ values of $\mathrm{CBT} /$ the $\mathrm{HU}$ values of TT) between $\mathrm{CBT}$ and TT was $1.92 \pm 0.46$. A significant difference was found between CBT and TT $(p=0.00, t=$ 72.25). The correlation coefficient between CBT and TT was 0.715 , which indicated a strong positive correlation. A significant difference was also presented in the HU values of CBT and TT and the specific value between CBT and TT between males and females (CBT: $p=0.00, t=8.996$; TT: $p=0.000, t=9.672$; CBT/TT: $p=0.024, t=2.269)$. The specific value of CBT between males and females increased from 1.08 \pm 0.27 in 20 to 29 to $1.23 \pm 0.31$ in above 70 , the specific value of TT between males and females increased from $1.12 \pm 0.32$ in 20 to 29 to $1.26 \pm 0.48$ in above 70, and the specific value of CBT/TT between males and females increased from $0.99 \pm 0.21$ in 20 to 29 to $1.05 \pm 0.27$ in above 70 .

For males, the $\mathrm{HU}$ values of CBT and TT were 384.75 \pm 103.44 and $211.71 \pm 64.74$, and the specific value between CBT and TT was $1.89 \pm 0.45$. In different decades, the specific value between CBT and TT increased from 
Table 1 Demographics of all specimens

\begin{tabular}{llllll}
\hline Age & Sex & Number & Mean age (years) & Mean height (cm) & Mean weight (kg) \\
\hline $20-29$ & Male & 20 & $24.27 \pm 3.12$ & $164.32 \pm 10.48$ & $63.14 \pm 5.17$ \\
& Female & 20 & $25.25 \pm 3.82$ & $157.24 \pm 7.14$ & $50.45 \pm 3.54$ \\
$30-39$ & Male & 20 & $33.82 \pm 2.54$ & $168.21 \pm 8.17$ & $66.78 \pm 4.23$ \\
& Female & 20 & $35.35 \pm 3.71$ & $160.74 \pm 7.98$ & $52.17 \pm 4.51$ \\
$40-49$ & Male & 20 & $44.88 \pm 1.88$ & $166.87 \pm 7.54$ & $70.79 \pm 6.21$ \\
& Female & 20 & $46.46 \pm 2.96$ & $161.28 \pm 6.19$ & $61.67 \pm 6.41$ \\
$50-59$ & Male & 20 & $54.06 \pm 3.84$ & $167.44 \pm 7.46$ & $69.18 \pm 7.83$ \\
& Female & 20 & $55.00 \pm 3.16$ & $159.97 \pm 7.17$ & $58.47 \pm 5.17$ \\
$60-69$ & Male & 20 & $64.37 \pm 2.47$ & $166.45 \pm 5.86$ & $64.14 \pm 7.41$ \\
& Female & 20 & $64.33 \pm 3.62$ & $160.47 \pm 5.24$ & $56.71 \pm 5.84$ \\
Above 70 & Male & 20 & $74.17 \pm 5.47$ & $158.15 \pm 6.17$ & $66.79 \pm 5.17$ \\
& Female & 20 & $74.00 \pm 4.13$ & $56.11 \pm 4.69$ \\
\hline
\end{tabular}

$1.65 \pm 0.26$ in 20 to 29 to $2.18 \pm 0.58$ in above 70 , and the correlation coefficient of the specific value between CBT and TT and decades was 0.99 . The correlation coefficient of the specific value between CBT and TT and different segments was 0.97. The specific value between CBT and TT increased from $1.59 \pm 0.29$ in L1 to $2.16 \pm 0.52$ in L5 (Figs. 2 and 4). A significant difference in specific value between CBT and TT values among different decades and different segments was observed (decades: $p=0.000$, $F=18.714$; segments: $p=0.000, F=32.400$; Table 3 ).

For females, the $\mathrm{HU}$ values of CBT and TT were $344.45 \pm 102.84$ and $185.56 \pm 67.61$, and the specific value between CBT and TT was $1.95 \pm 0.47$. In different decades, the specific value between CBT and TT increased from $1.72 \pm 0.35$ in 20 to 29 to $2.11 \pm 0.39$ in above 70. However, females demonstrated an increasing trend with a notable inconsistency in ages 40 to $49(R=0.94)$. The correlation coefficient of the specific value between CBT and TT and different segments was 0.99. The specific value between CBT and $\mathrm{TT}$ also increased from $1.64 \pm 0.33$ in $\mathrm{L} 1$ to $2.27 \pm$ 0.56 in L5 (Figs. 3 and 4). A significant difference in the specific value between CBT and TT values among different decades and segments was observed (decades: $p=0.000, F=8.688$; segments: $p=0.000, F=$ 37.313; Table 4).

\section{Discussion}

Pedicle screw fixation with the traditional convergent trajectory has been a gold standard technique for spinal

Table $2 \mathrm{HU}$ values at the trajectory of CBT and $\Pi \mathrm{T}$ in different decades

\begin{tabular}{|c|c|c|c|c|c|c|c|c|}
\hline & Sex & Total & $20-29$ & $30-39$ & $40-49$ & $50-59$ & $60-69$ & Above 70 \\
\hline \multirow[t]{3}{*}{ CBT } & M & $384.75 \pm 103.44$ & $454.75 \pm 83.39^{* \Delta \ddagger} \epsilon$ & $432.14 \pm 74.22^{+\cdot \varepsilon}$ & $405.15 \pm 85.45^{9}$ & $379.08 \pm 104.15^{\varnothing \psi}$ & $336.86 \pm 107.88$ & $306.65 \pm 76.67$ \\
\hline & $\mathrm{F}$ & $344.45 \pm 102.84$ & $432.68 \pm 77.80^{* \Delta \ddagger} \epsilon$ & $401.93 \pm 89.90^{+\cdot £}$ & $363.64 \pm 75.76^{\wedge я}$ & $344.20 \pm 116.75^{\varnothing \psi}$ & $289.62 \pm 63.70$ & $257.76 \pm 59.71$ \\
\hline & T & $365.19 \pm 105.05$ & $444.37 \pm 81.33^{* \Delta \ddagger} \epsilon$ & $417.03 \pm 83.57^{※+\cdot \varepsilon}$ & $387.16 \pm 83.71^{\wedge A}$ & $361.64 \pm 111.68^{\varnothing 4}$ & $313.87 \pm 92.03^{\sim}$ & 281.5 \\
\hline \multirow[t]{3}{*}{$\pi$} & M & $211.71 \pm 64.74$ & $278.56 \pm 47.30^{* \Delta \neq \epsilon}$ & $254.75 \pm 55.55^{\dagger \cdot \pm}$ & $225.40 \pm 38.08^{\wedge я}$ & $197.18 \pm 45.05^{\varnothing 4}$ & $170.64 \pm 51.05^{\sim}$ & $148.36 \pm 44.44$ \\
\hline & $\mathrm{F}$ & $185.56 \pm 67.61$ & $257.07 \pm 47.94^{\# * \Delta \neq \epsilon}$ & $214.94 \pm 52.67^{\dagger • £}$ & $209.79 \pm 77.45^{\S \wedge Я}$ & $182.80 \pm 54.90^{\varnothing \Psi}$ & $143.32 \pm 34.67$ & $124.98 \pm 31.23$ \\
\hline & $\mathrm{T}$ & $199.02 \pm 67.89$ & $268.44 \pm 48.6^{6 \# * \Delta \ddagger \epsilon}$ & $234.84 \pm 57.54 \%+• £$ & $218.64 \pm 58.77^{\S \wedge Я}$ & $189.99 \pm 50.59^{\varnothing \psi}$ & $157.35 \pm 45.839^{\sim}$ & $136.35 \pm .9 .8$ \\
\hline
\end{tabular}

Statistical significance $(p<0.05)$

CBT cortical bone trajectory, $T$ traditional trajectory, $M$ males, $F$ females, $T$ total

\#20-29 versus 30-39 significant difference

*20-29 versus 40-49 significant difference

${ }^{\Delta} 20-29$ versus $50-59$ significant difference

${ }^{*} 20-29$ versus $60-69$ significant difference

$\epsilon_{20-29}$ versus over 70 significant difference

※30-39 versus $40-49$ significant difference

† $30-39$ versus $50-59$ significant difference

-30-39 versus $60-69$ significant difference

$50-39$ versus over 70 significant difference

$\$ 40-49$ versus $50-59$ significant difference

$\wedge 40-49$ versus $60-69$ significant difference

${ }^{9} 40-49$ versus over 70 significant difference

$\varnothing_{50-59}$ versus $60-69$ significant difference

${ }_{50}$-59 versus over 70 significant difference

60-69 versus over 70 significant difference 


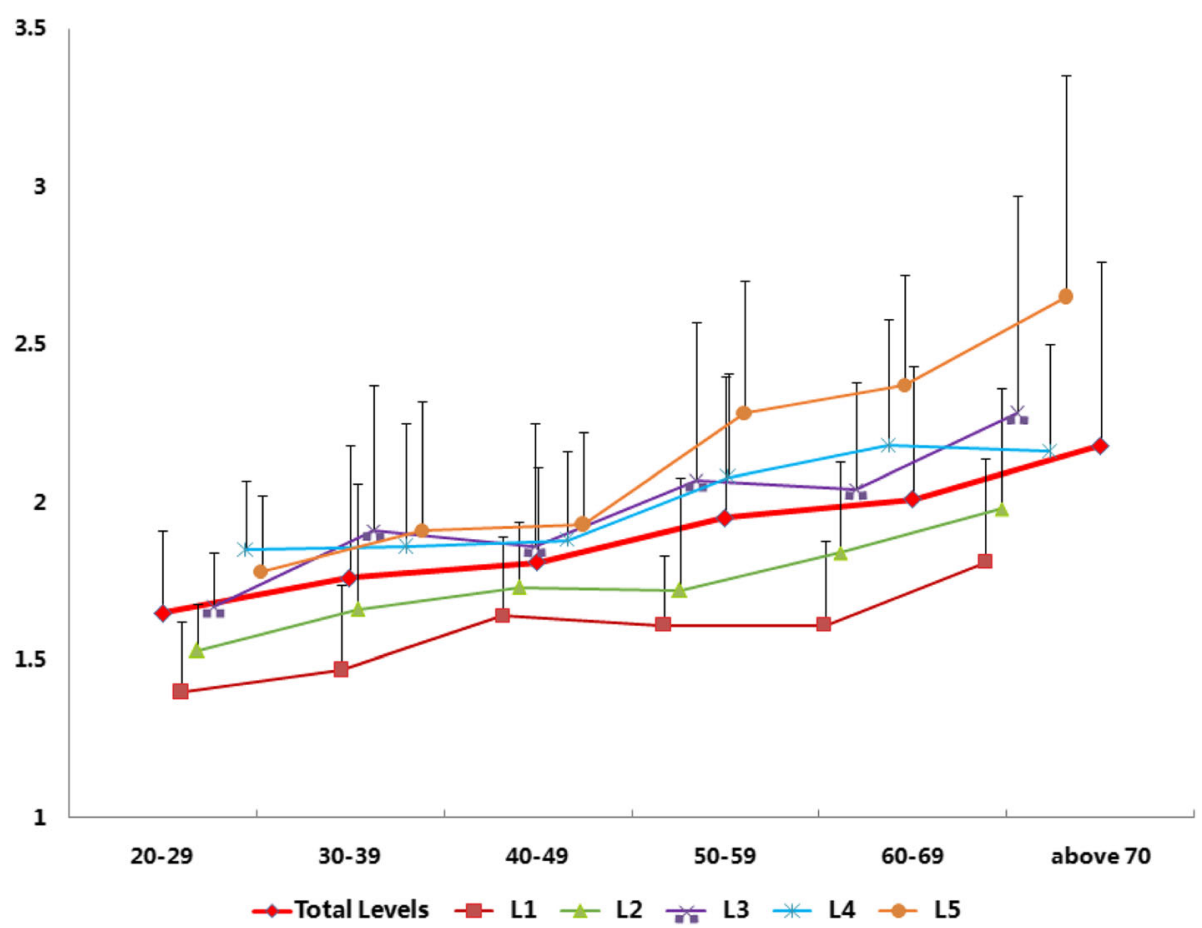

Fig. 2 Trend of the specific value between CBT and TT (+ s.d.) of different lumbar levels in males. The average specific value between CBT and $T$ $(+$ s.d.) increased with increasing age and cauda lumbar level

fixation. Although this technique has stood the test of time, it does have limitations, particularly in elderly osteoporotic and obese patients. With the traditional convergent trajectory, most of the screw thread surface rests in medullary bone, hence becoming prone to screw loosening secondary to age-related changes in bone quality and providing inconsiderably rigid fixation [7]. In obese patients with an increased depth of operating corridor, achieving a tension-free screw insertion necessitates a long incision with further lateral dissection with consequent increased blood loss, the risk of injury to the innervation of facet joints, and multifidus muscle. These limitations could potentially be overcome with a divergent trajectory (CBT) and a medial starting point. Many studies have demonstrated that the new CBT has equivalent pullout and toggle characteristics compared with the TT $[7,9,11,17,18]$.

Several studies have correlated BMD with traditional pedicle pullout strength $[8,19]$. HUs have been used to approximate regional oral BMD and have been shown to correlate strongly with insertion torque and the stability of metal implants in vitro and in vivo [13-15].

Table 3 Specific value between CBT and $\Pi$ in different segments on different decades of males

\begin{tabular}{|c|c|c|c|c|c|c|c|}
\hline Segment/CBT/TT & Total & $20-29$ & $30-39$ & $40-49$ & $50-59$ & $60-69$ & Above 70 \\
\hline Total & $1.89 \pm 0.45$ & $1.65 \pm 0.26$ & $1.76 \pm 0.42$ & $1.81 \pm 0.30$ & $1.95 \pm 0.45$ & $2.01 \pm 0.42$ & $2.18 \pm 0.58$ \\
\hline L1 & $1.59 \pm 0.29^{\# * \Delta \neq}$ & $1.40 \pm 0.22^{* \Delta \ddagger}$ & $1.47 \pm 0.27^{* \Delta \ddagger}$ & $1.64 \pm 0.25^{\ddagger}$ & $1.61 \pm 0.22^{* \Delta \ddagger}$ & $1.61 \pm 0.27^{* \Delta \neq}$ & $1.81 \pm 0.33$ \\
\hline L2 & $1.75 \pm 0.34^{\epsilon+\bullet}$ & $1.53 \pm 0.15^{t \cdot}$ & $1.66 \pm 0.40$ & $1.73 \pm 0.21$ & $1.72 \pm 0.36^{\circ}$ & $1.84 \pm 0.29^{+}$ & $1.98 \pm 0.38$ \\
\hline L3 & $1.97 \pm 0.48^{f}$ & $1.67 \pm 0.17$ & $1.91 \pm 0.46$ & $1.86 \pm 0.39$ & $2.07 \pm 0.50$ & $2.04 \pm 0.34^{ \pm}$ & $2.28 \pm 0.69$ \\
\hline$\llcorner 4$ & $2.01 \pm 0.36$ & $1.85 \pm 0.22$ & $1.86 \pm 0.39$ & $1.88 \pm 0.28$ & $2.08 \pm 0.33$ & $2.18 \pm 0.40$ & $2.16 \pm 0.34$ \\
\hline L5 & $2.16 \pm 0.52$ & $1.78 \pm 0.24$ & $1.91 \pm 0.41$ & $1.93 \pm 0.29$ & $2.28 \pm 0.42$ & $2.37 \pm 0.35$ & $2.65 \pm 0.70$ \\
\hline
\end{tabular}

Statistical significance $(p<0.05)$

\#L1 versus L2 significant difference

* $L 1$ versus $L 3$ significant difference

$\Delta L 1$ versus $L 4$ significant difference

$\mathrm{L} L 1$ versus $\mathrm{L} 5$ significant difference

$\epsilon$ 2 versus $L 3$ significant difference

${ }^{+} L 2$ versus $L 4$ significant difference

$L 2$ versus $L 5$ significant difference

${ }^{E} L 3$ versus $L 5$ significant difference 


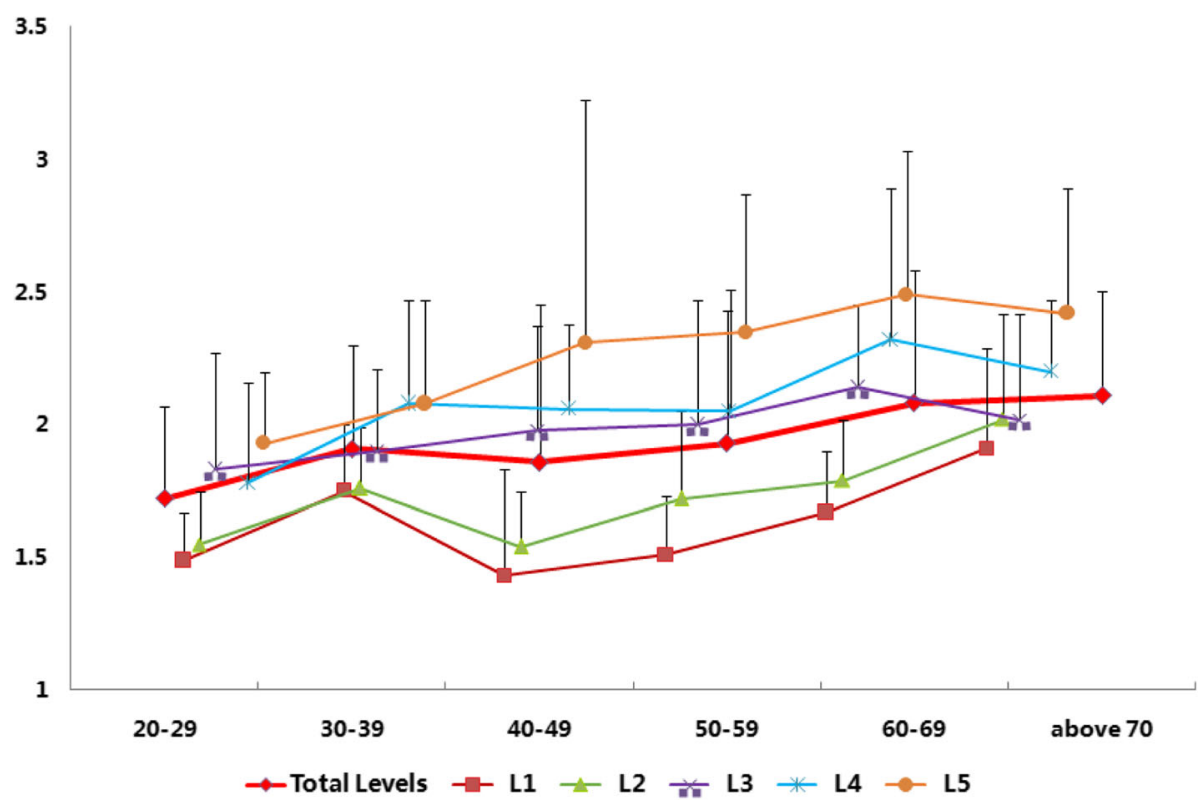

Fig. 3 Trend of the specific value between CBT and TT (+ s.d.) of the different lumbar levels in females. Female patients experienced a notable decrease during their ages of 40 to 49 , which corresponds to the age of menopause. The average specific value between CBT and $\Pi T$ (+ s.d.) increased with cauda lumbar level

Therefore, we measured the $\mathrm{HU}$ values of the ROI of each trajectory to represent the BMD. While BMD has historically been assessed using dual-energy X-ray absorptiometry (DXA) and quantitative CT (qCT), the HU values from $\mathrm{CT}$ scans offer several benefits. HU values can be obtained throughout the entire spine, where DXA standards have not been defined. DXA results can be artificially elevated by lumbar scoliosis and degenerative changes [20, 21]. Other advantages of HU assessment over DXA assessment include the fact that $\mathrm{HU}$ assessment represents 3D BMD and that it can be localized to any ROI to represent the ideal trajectory; meanwhile, DXA can only measure the BMD of vertebrae, which cannot represent the trajectory. qCT examines that the bone density is the cancellous bone separate from the cortical bone in vertebrae [22-24], and the ROI of this method cannot represent the specific trajectory that combines cortical and cancellous bones. Thus, $\mathrm{HU}$ values can be useful in medical and surgical management.

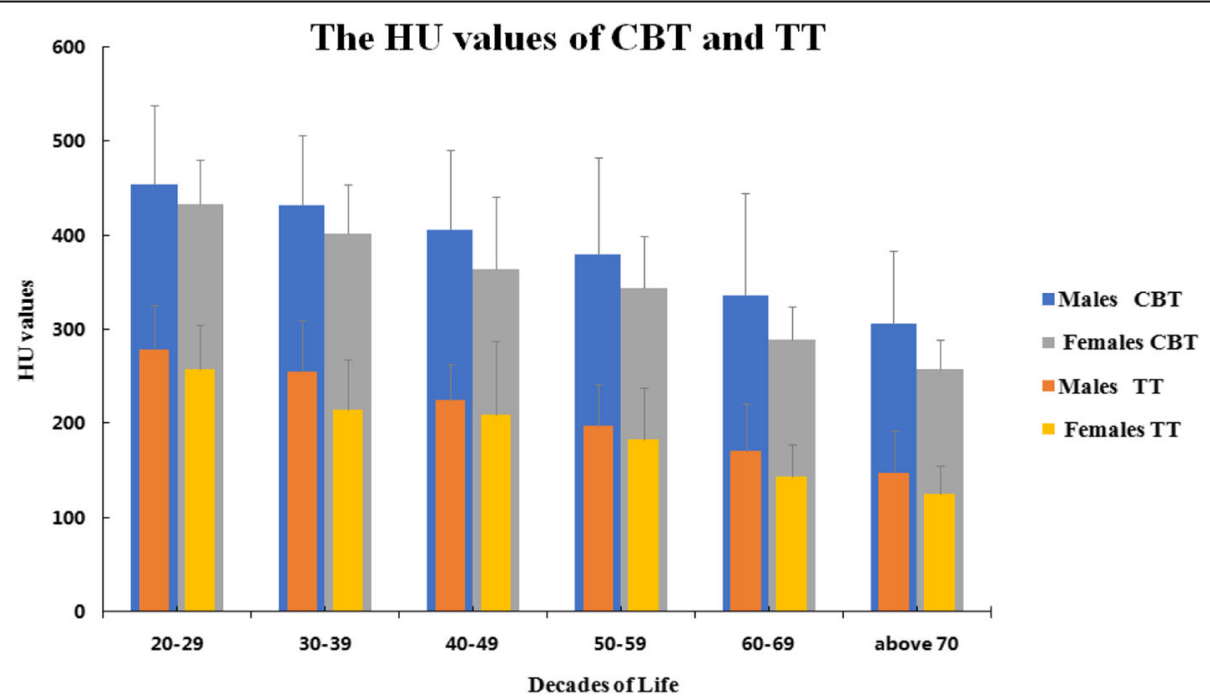

Fig. $4 \mathrm{HU}$ values of CBT and $T \mathrm{~T}$ in different decades between males and females (+ s.d.). The HU values of CBT were larger than those of $\mathrm{TT}$ in males and females. The $\mathrm{HU}$ values of CBT and $T T$ decreased with increasing age 
Table 4 Specific value between CBT and $\pi$ in different segments on different decades of females

\begin{tabular}{llllllll}
\hline Segment/CBT/TT & Total & $20-29$ & $30-39$ & $40-49$ & $50-59$ & $60-69$ & Above 70 \\
\hline Total & $1.95 \pm 0.47$ & $1.72 \pm 0.35$ & $1.91 \pm 0.39$ & $1.86 \pm 0.59$ & $1.93 \pm 0.50$ & $2.08 \pm 0.50$ & $2.11 \pm 0.39$ \\
L1 & $1.64 \pm 0.33^{* \Delta \neq}$ & $1.49 \pm 0.18^{*^{\ddagger}}$ & $1.75 \pm 0.25$ & $1.43 \pm 0.40^{\Delta \neq}$ & $1.51 \pm 0.22^{* \Delta \neq}$ & $1.67 \pm 0.23^{* \Delta \neq}$ & $1.91 \pm 0.38^{\ddagger}$ \\
L2 & $1.74 \pm 0.32^{\epsilon+\cdot}$ & $1.55 \pm 0.20^{*}$ & $1.76 \pm 0.23$ & $1.54 \pm 0.21^{*}$ & $1.72 \pm 0.33^{*}$ & $1.79 \pm 0.23^{\dagger \cdot}$ & $2.02 \pm 0.40^{*}$ \\
L3 & $1.98 \pm 0.36^{ \pm}$ & $1.83 \pm 0.44$ & $1.90 \pm 0.31$ & $1.98 \pm 0.39$ & $2.00 \pm 0.47$ & $2.14 \pm 0.31$ & $2.02 \pm 0.40^{ \pm}$ \\
L4 & $2.09 \pm 0.47^{\&}$ & $1.78 \pm 0.38$ & $2.08 \pm 0.39$ & $2.06 \pm 0.32$ & $2.05 \pm 0.46$ & $2.32 \pm 0.57$ & $2.20 \pm 0.27$ \\
L5 & $2.27 \pm 0.56$ & $1.93 \pm 0.27$ & $2.08 \pm 0.39$ & $2.31 \pm 0.91$ & $2.35 \pm 0.52$ & $2.49 \pm 0.54$ & $2.42 \pm 0.47$ \\
\hline
\end{tabular}

\#L1 versus $L 2$ significant difference

* L1 versus L3 significant difference

${ }^{\Delta} \mathrm{L} 1$ versus $\mathrm{L} 4$ significant difference

${ }^{\ddagger} \mathrm{L} 1$ versus $\mathrm{L} 5$ significant difference

$\epsilon$ L2 versus $L 3$ significant difference

${ }^{t} L 2$ versus $L 4$ significant difference

$L 2$ versus $L 5$ significant difference

${ }^{\mathbf{L}} \mathrm{L} 3$ versus $L 5$ significant difference

${ }^{\&}$ L4 versus L5 significant difference

Wray et al. illustrated that an increased bone quality was present in CBT trajectory compared with that in TT in their biomechanical study and concluded that the CBT represented a good option to obtain fixation for the lumbar spine with low-quality bone [17]. Our study kept with this conclusion from an iconography using $\mathrm{HU}$ values. In our study, we found that the mean $\mathrm{HU}$ values were $365.19 \pm$ 105.05 on CBT and $199.02 \pm 67.89$ on TT, and the specific value between CBT and TT was $1.92 \pm 0.46$. Previous studies have reported a significant linear correlation between increasing age and decreasing BMD (evaluated by $\mathrm{HU}$ values) at the lumbar mid-vertebral body [12]. We also determined that the HU values of CBT and TT decreased with the increase of age. Nevertheless, the specific value between the $\mathrm{HU}$ values of CBT and TT increased, which may be attributed to osteoporosis more disproportionately affecting the trabecular bone than the cortical bone; thus, the fixation achieved within the vertebral body was compromised [24], and it could be explained by the fact that the CBT screws being anchored mainly in cortical bone are less influenced by the osteoporotic process than the traditional screws in cancellous bone [8].

In our study, a significant difference was observed in the HU values between male and female patients and different decades for the CBT and TT groups. Kojima et al. [16] also observed this phenomenon. However, his sub-group of decades was only over and under the age of 70 years. Female patients over the age of 70 years presented the lowest mean HU values within the ROI for CBT and TT. Mai et al. [25] concluded that the osteoporosis cohort demonstrated a significantly greater difference in $\mathrm{HU}$ values between CBT and TT fixation points compared with that of the non-osteoporotic cohort at all lumbar levels. The previous study also determined that the relative differences in $\mathrm{HU}$ values significantly increased with each decade of age at each lumbar spinal level. Furthermore, we found that the $\mathrm{HU}$ values of lower lumbar vertebrae were larger than those of upper lumbar vertebrae, which might be explained by the fact that cauda lumbars mostly bear the weight of activities.

Females aged 40 to 49 demonstrated an increasing trend with a notable inconsistency. The reason for this phenomenon might be the age of menopause. Similarly, Mai et al. [25] found that the relative increase in BMD between CBT and TT fixation points exhibited a notable decrease in menopause females. Schreiber et al. [26] also reported a decrease in HU-determined BMD of the lumbar vertebral bodies in females around the age of menopause.

The pedicle widths of L1 and L2 were often smaller than the ROI, which might indicate that placing CBT screws in L1 and L2 is difficult, as we also found in our previous morphometric study. Ohkawa [18] reported that the misplacing rate was approximately $4 \%$ using the CBT technique. Thus, surgeons should advance the CBT screw slowly using a $\mathrm{C}$ arm or CT image-guided navigation to confirm its accurate placement, and many other studies have also suggested using some tools to avoid complications [8, 27, 28].

Our study has some limitations. First, we performed our study with the Chinese. Further study should be performed to analyze whether a difference exists among human races when using CBT screws. Second, we used the ROI to represent the ideal trajectory for CBT and TT. However, actual surgical technique and trajectory may differ depending on the surgeon.

\section{Abbreviations \\ BMD: Bone mineral density; CBT: Cortical bone trajectory; CT: Computed tomography; DXA: Dual-energy X-ray absorptiometry; HUs: Hounsfield units; qCT: Quantitative CT; ROI: Region of interest; $T$ : Traditional trajectory}

\section{Acknowledgements}

Not applicable.

\section{Funding}

This work is supported by the University Nature Scientific Research Project of Anhui Province [grant number KJ2017A832], Program for Science and 
Technology Development of Anhui Province [grant number 1704a0802158], and the Natural science foundation project of Anhui Province [grant number 1408085QH174].

\section{Availability of data and materials}

The datasets used and/or analysed during the current study are available from the corresponding author on reasonable request.

\section{Authors' contributions}

$\mathrm{CLS}, \mathrm{HG}, \mathrm{HML}$, and RJZ conceived and coordinated the study, designed, performed, and analyzed the experiments, and wrote the paper. TX and CYJ carried out the data collection and data analysis and revised the paper. JXZ and FLD designed the study, carried out the data analysis, and revised the paper. All authors reviewed the results and approved the final version of the manuscript.

\section{Ethics approval and consent to participate}

Not applicable.

\section{Consent for publication}

Not applicable.

\section{Competing interests}

The authors declare that they have no competing interests.

\section{Publisher's Note}

Springer Nature remains neutral with regard to jurisdictional claims in published maps and institutional affiliations.

\section{Author details}

'Department of Orthopedics, The First Affiliated Hospital of Anhui Medical University, 210 Jixi Road, Hefei 230022, Anhui, China. ${ }^{2}$ Department of Orthopedics, The First Affiliated Hospital of USTC (Anhui Provincial Hospital), Hefei 230022, Anhui, China. ${ }^{3}$ Department of Orthopedics and Spine Surgery, The First Affiliated Hospital of Anhui Medical University, Hefei 230022, Anhui, China.

\section{Received: 25 February 2019 Accepted: 26 April 2019}

\section{Published online: 09 May 2019}

\section{References}

1. Hart RA, Hansen BL, Shea M, Hsu F, Anderson GJ. Pedicle screw placement in the thoracic spine: a comparison of image-guided and manual techniques in cadavers. Spine. 2005;30(12):E326-31.

2. Wittenberg RH, Shea M, Swartz DE, Lee KS, White AA, Hayes WC Importance of bone mineral density in instrumented spine fusions. Spine. 1991:16(6):647-52.

3. Cook SD, Salkeld SL, Stanley T, Faciane A, Miller SD. Biomechanica study of pedicle screw fixation in severely osteoporotic bone. Spine J. 2004;4(4):402-8

4. Davne SH, Myers DL. Complications of lumbar spinal fusion with transpedicular instrumentation. Spine. 1992;17(6 Suppl):184-9.

5. Okuyama K, Abe E, Suzuki T, Tamura Y, Chiba M, Sato K. Influence of bone mineral density on pedicle screw fixation: a study of pedicle screw fixation augmenting posterior lumbar interbody fusion in elderly patients. Spine J. 2001;1(6):402-7.

6. Suzuki T, Abe E, Okuyama K, Sato K. Improving the pullout strength of pedicle screws by screw coupling. J Spinal Disord. 2001:14(5):399.

7. Santoni BG, Hynes RA, McGilvray KC, Rodriguez-Canessa G, Lyons AS, Henson MAW, Womack WJ, Puttlitz CM. Cortical bone trajectory for lumbar pedicle screws. Spine J. 2009;9(5):366-73.

8. Matsukawa K, Yato Y, Kato T, Imabayashi H, Asazuma T, Nemoto K. In vivo analysis of insertional torque during pedicle screwing using cortical bone trajectory technique. Spine. 2014;39(4):E240-5.

9. Baluch DA, Patel AA, Lullo B, Havey RM, Voronov LI, Nguyen N-L, Carandang G, Ghanayem AJ, Patwardhan AG. Effect of physiological loads on cortical and traditional pedicle screw fixation. Spine. 2014;39(22):E1297-302.

10. Crookshank M, Ploeg HL, Ellis RE, Macintyre NJ. Repeatable calibration of Hounsfield units to mineral density and effect of scanning medium. Advances in Biomechanics and Applications. 2013;1(1):15-22.
11. Eswaran SK, Gupta A, Adams MF, Keaveny TM. Cortical and trabecular load sharing in the human vertebral body. J Bone Miner Res. 2006;21(2):307-14.

12. Schreiber JJ, Anderson PA, Rosas HG, Buchholz AL, Au AG. Hounsfield units for assessing bone mineral density and strength: a tool for osteoporosis management. J Bone Joint Surg Am. 2011;93(11):1057-63.

13. Ilser T, Lars S, Mcglumphy EA, Tözüm TF. Biomechanical aspects of primary implant stability: a human cadaver study. Clin Implant Dent Relat Res. 2009; 11(2):113.

14. Turkyilmaz I, Tumer C, Ozbek EN, Tözüm TF. Relations between the bone density values from computerized tomography, and implant stability parameters: a clinical study of 230 regular platform implants. J Clin Periodontol. 2007;34(8):716-22.

15. Turkyilmaz I, Aksoy U, Mcglumphy EA. Two alternative surgical techniques for enhancing primary implant stability in the posterior maxilla: a clinical study including bone density, insertion torque, and resonance frequency analysis data. Clin Implant Dent Relat Res. 2008;10(4):231.

16. Kojima K, Asamoto S, Kobayashi Y, Ishikawa M, Fukui Y. Cortical bone trajectory and traditional trajectory--a radiological evaluation of screw-bone contact. Acta Neurochir. 2015:157(7):1173-8.

17. Steven W, Ronnie M, Sasidhar V, Shetye SS, Mcgilvray KC, Puttlitz CM. Pedicle screw placement in the lumbar spine: effect of trajectory and screw design on acute biomechanical purchase. J Neurosurg Spine. 2015;22(5): 503-10

18. Ohkawa T, Iwatsuki K, Ohnishi Y, Ninomiya K, Yoshimine T. Isthmus-guided cortical bone trajectory reduces postoperative increases in serum creatinine phosphokinase concentrations. Orthop Surg. 2015;7(3):232-8.

19. Lee JH, Lee JH, Park JW, Shin YH. The insertional torque of a pedicle screw has a positive correlation with bone mineral density in posterior lumbar pedicle screw fixation. J Bone Joint Surg Br. 2012;94(1):93.

20. Pappou IP, Girardi FP, Sandhu HS, Parvataneni HK, Jr CF, Schneider R, Frelinghuysen $\mathrm{P}$, Lane JM. Discordantly high spinal bone mineral density values in patients with adult lumbar scoliosis. Spine. 2006;31(14):1614-20.

21. Rand T, Seidl G, Kainberger F, Resch A, Hittmair K, Schneider B, Glüer CC, Imhof $\mathrm{H}$. Impact of spinal degenerative changes on the evaluation of bone mineral density with dual energy X-ray absorptiometry (DXA). Calcif Tissue Int. 1997;60(5):430-3.

22. Overton TR. Quantitative computed tomography. Acta Orthop Scand. 1989: 60(5):629-30.

23. Adams JE. Quantitative computed tomography. Eur J Radiol. 2009;71(3):415.

24. Richardson ML, Genant HK, Cann CE, Ettinger B, Gordan GS, Kolb FO, Reiser UJ. Assessment of metabolic bone diseases by quantitative computed tomography. Clin Orthop Relat Res. 1985;(195):224-38.

25. Mai HT, Mitchell SM, Hashmi SZ, Jenkins TJ, Patel AA, Hsu WK. Differences in bone mineral density of fixation points between lumbar cortical and traditional pedicle screws. Spine J. 2016;16(7):835-41.

26. Schreiber JJ, Anderson PA, Hsu WK. Use of computed tomography for assessing bone mineral density. Neurosurg Focus. 2014;37(1):E4.

27. Tortolani PJ, Stroh DA. Cortical bone trajectory technique for posterior spinal instrumentation. J Am Acad Orthop Surg. 2016;24(11):755-61.

28. Rodriguez A, Neal MT, Liu A, Somasundaram A, Hsu W, Jr BC. Novel placement of cortical bone trajectory screws in previously instrumented pedicles for adjacent-segment lumbar disease using CT image-guided navigation. Neurosurg Focus. 2014;36(3):E9.

Ready to submit your research? Choose BMC and benefit from:

- fast, convenient online submission

- thorough peer review by experienced researchers in your field

- rapid publication on acceptance

- support for research data, including large and complex data types

- gold Open Access which fosters wider collaboration and increased citations

- maximum visibility for your research: over $100 \mathrm{M}$ website views per year

At $\mathrm{BMC}$, research is always in progress.

Learn more biomedcentral.com/submission 